\title{
The WSN Energy Efficient Protocol Based on Adaptive Mechanism of Sleep
}

\author{
Liu Changzheng and Zhang Ronghua*
}

College of Information Science and Technology, Shihezi University, Shihezi, 832003, China

\begin{abstract}
In view of node energy limitation in the wireless sensor network, efficient energy saving strategy has become one of the hot spots in the wireless sensor technology. Through the analysis of the energy efficiency solution of wireless sensor network in each layer, put forward a kind of wireless sensor energy efficient protocol based on adaptive sleep mechanism. The scheme according to the residual energy storage, location and degree of adjacent nodes load, using variable duration of sleep mechanism to choose forward grouping way. Compared with the random transmission scheme based on the geographical position, the given scheme can save the network node energy greatly and balance the network energy consumption effectively. Therefore, this scheme can improve overall network life significantly.
\end{abstract}

Keywords: Adaptive mechanism of sleep, energy efficiently, random forward, wireless sensor network.

\section{INTRODUCTION}

Wireless Sensor Network (WSN) is due to the microelectronics technology, computer technology and wireless communication technology rapid development combining of the product [1]. Through randomly deployed a large number of low-cost micro sensor nodes in monitoring area, then adopts the wireless communication technology form a selforganization network system based on multiple hops routing. Its main function is using the sensor node collaboration awareness, collection and processing network coverage within the scope of the monitoring object information, which is used widely in military, medical, transportation, agriculture and environmental monitoring, etc. Wireless sensor network node has the advantages of small volume and low price, through the plane spreading or artillery scatters practices such as distribution in harsh environment which unsuited for exploration area. However, wireless sensor network exist nodes power energy limited, communication ability limited, computing storage capacity limited and so on shortcomings at the same time. Among of them, the sensor node energy consumption too fast and the network energy consumption too unbalanced become the main factors restriction life of wireless sensor network, is also an important bottleneck which affecting the development of wireless sensor network. Therefore, how to improve the energy efficiency and save energy consumption has become a key point and hot issues in the research of wireless sensor network.

\section{THE ENERGY SAVING STRATEGY OF EACH LAYER OF THE WSN SYSTEM ARCHITECTURE}

Due to the life cycle of wireless sensor network rely on batteries energy of sensor nodes, however, the energy of the

*Address correspondence to this author at the College of Information Science and Technology, Shihezi University, Shihezi, 832003, China; Tel: +86993 2058090; E-mail: zrh_oea@sina.com sensor node is very limited, once the battery is depleted, to charge or change batteries node is generally not reality [2]. So it needs to consider the protocol stack of wireless sensor network at all levels and takes appropriate energy efficient protocol design strategy. At present due to the application system of wireless sensor network is not the same, so it appears different architecture models. Fig. (1) shows the typical system architecture of wireless sensor network protocol stack model [3] and energy optimization strategy of each layer. Due to the resources of the sensor nodes and calculate storage capacity limited, generally considered only in the physical layer, data link layer and network layer three levels of energy efficient protocol design strategy research.

\section{TYPICAL ENERGY EFFICIENT PROTOCOL ANALYSIS}

In order to improve energy utilization efficiency of wireless sensor network, put forward variety of effective energy efficient protocol in view of the wireless sensor network protocol stack at all levels. Literature [4] proposes a multistage forwarding method based on energy consumption level in the physical layer, which can consume energy according to different needs, reduce the waste of energy, but it requires more complex hardware support. Literature [5] uses regular sleep mechanism to control the sensor node energy consumption in the data link layer, however, which likely to make the network load aggravating, cause other network node energy waste. Literature [6] is a kind of the MAC frame address shorten of the protocol design, reduce the energy consumption in sending data, but it likely to result in heterogeneous network incompatible. In the network layer, literature [7] presents a routing protocol based on energy consciousness, through the surplus store energy according to node in the network routing, the choice of the forward routing is not necessarily the best routing, would be likely to cause the overall network energy consumption increase. Literature [8] through establishing multiple alternate routing, according to the probability to choose size, which can avoid 
protocol stack architecture model

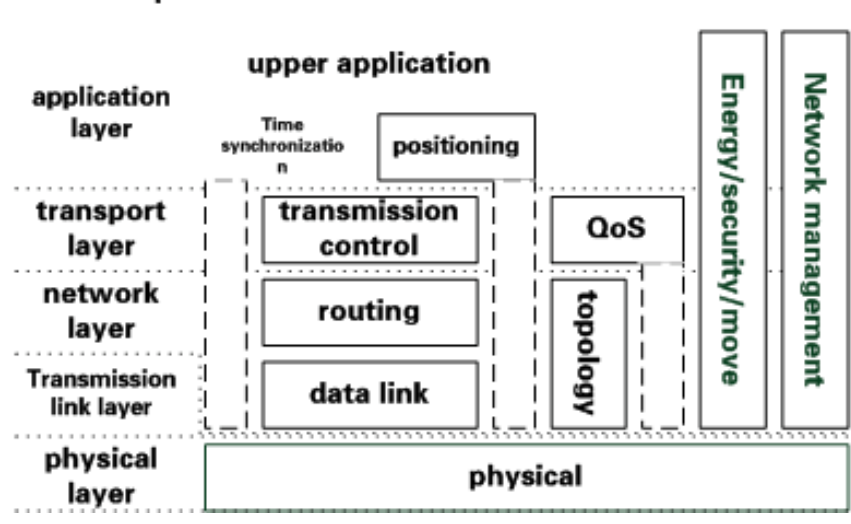

Energy efficient strategy

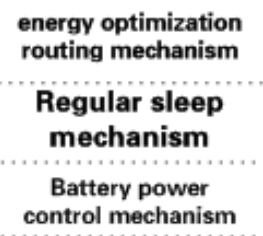

Fig. (1). Wireless sensor network protocol stack and energy efficient strategy.

Table 1. Energy efficient advantages and disadvantages of wireless sensor network protocol stack layer.

\begin{tabular}{|c|c|c|c|}
\hline Protocol Stack Layer & Typical Energy Efficient Protocol & Advantages & Disadvantages \\
\hline \hline Network layer & $\begin{array}{c}\text { Routing protocol based on energy } \\
\text { consciousness; } \\
\text { alternate routing probability for- } \\
\text { ward agreement }\end{array}$ & $\begin{array}{c}\text { According to the residual energy of } \\
\text { nodes in the routing choice; } \\
\text { according to the probability to choose } \\
\text { routing, avoid energy "empty hole" }\end{array}$ & $\begin{array}{c}\text { Choice is not the best route, increase the overall } \\
\text { network energy consumption; } \\
\text { frequently choose cluster nodes increases the } \\
\text { energy consumption }\end{array}$ \\
\hline Data link layer & $\begin{array}{c}\text { Mechanism protocol based on the } \\
\text { regular sleep; } \\
\text { MAC frame address shorten } \\
\text { protocol }\end{array}$ & $\begin{array}{c}\text { Save the sensor node energy; } \\
\text { reduce energy consumption of send- } \\
\text { ing data }\end{array}$ & $\begin{array}{c}\text { Network load increasing, energy consumption } \\
\text { imbalanced; }\end{array}$ \\
\hline Physical layer & Multi-layer energy forward protocol & $\begin{array}{c}\text { According to different forward and } \\
\text { demand consumption energy }\end{array}$ & $\begin{array}{c}\text { Need complex hardware support } \\
\text { patible }\end{array}$ \\
\hline $\begin{array}{c}\text { Cross layer } \\
\text { optimization }\end{array}$ & $\begin{array}{c}\text { Cross-layer energy optimization } \\
\text { protocol }\end{array}$ & $\begin{array}{c}\text { Ensure the end-to-end transmission } \\
\text { probability of success }\end{array}$ & $\begin{array}{c}\text { Need to consider the details more, implementa- } \\
\text { tion is more complicated }\end{array}$ \\
\hline
\end{tabular}

the network energy consumption of a region focus formation energy "empty hole", but often choose cluster nodes makes communication energy consumption spending increases.

In addition, the difference of wireless sensor network and traditional network dividing layer is protocol stack work closely with each other and information sharing among the layers, so it should not cling to traditional hierarchical design concept, but to optimize the network performance by crosslayer design [9]. Now, researchers have proposed many effective across levels of energy efficient protocol. For example, F. BOUABDALLAH put forward the physical layer, data link layer and network layer of joint energy optimization agreement, which can guarantee the transmission probability of success, but need to consider the details more, and protocol implementation is more complicated in the literature [10]. Among them, it presented the wireless sensor network of all levels of energy efficient protocol advantages and disadvantages compared in Table 1.

\section{THE ENERGY EFFICIENT PROTOCOL BASED ON ADAPTIVE SLEEP MECHANISM}

ZORZI M. puts forward the forwarding protocol based on the geographical position of random (Geographical Ran- dom Forwarding Protocol, referred to as "GERAF Protocol") in the literature [11], which according to the geographical location information by jumping to choose the best nodes forward data packets. It is simple in implementation and has the advantages of strong robustness for the structural change of network topology. But it did not consider the node residual energy problems. Because of limited resources of wireless sensor network, to reduce the network energy consumption and prolong the network life is the two important goals of wireless sensors. In view of the implementation situation based on the geographical position in random forwarding agreement did not consider the residual stored energy imbalance of each node, we presents a mechanism of energy efficient protocol based on adaptive sleep (Energy-efficiency Adaptive Sleeping Protocol, referred to as "E2AS Protocol"). It choose the best forward path according to the node energy and distance factor, set reasonable longer sleep time at the same time according to the remaining stored energy and the adjacent node load, which can reduce the node energy consumption, balance network energy consumption and prolong the whole network life.

Due to the topology structure of the wireless sensor network will be dynamic changes as nodes into sleep mode, which can make the network routing choice and node sleep 
mechanism influence each other, but it is necessary to implement energy efficient. Thus there are three key problems need to be considered: (1) What nodes can enter the sleep state; (2) Under what circumstances nodes can sleep; (3) How to determine the nodes sleep duration time. The protocol design description based on adaptive mechanism of sleep energy efficient is given below.

Assume that all nodes are in sleep, and have the initial fixed energy. Nodes sleep time is random, part of the node activation into the listening state at the same time, the monitored data channel and busy tone of channel time is $T_{l}$. RTP shows the request forwarding packet, CTP shows the response request packet.

1) If the node receives the RTP packet in $T_{l}$ time, the node into receiving state, and according to the formula (1) to determine the node send time slot.

2) If the node send only a CTP response group, then the node will forward the data packet, do not transmit to other nodes group.

3) Grouping needs to send multiple CTP response within the same time slot, the repeater machine will send collision packet, none forwarding group will not be allowed to forward, is forwarding until the end of the collision of grouping to forward further.

4) Before the next time slot arrival, if it don't need to send the CTP group respond request, then expected to calculate time slot for the current time slot of the other place at the receiving node send CTP response group, finally select a node forwarding group.

Here, the nodes received time slot size is calculated according to the node energy consumption when the nodes send CTP group, the less energy of nodes has been consumed, the more likely to get forward time slot. The node $N_{i}$ has consumed energy $C_{i}$ of calculation formula is:

$$
C_{i}=\frac{r D_{i}}{T_{i} E_{i}}
$$

where $r$ shows the balance factor, $D_{i}$ shows the distance to the receiving node, $E_{i}$ shows residual energy of node $N_{i}$, $T_{i}$ shows the work time as forwarding nodes.

1) If the node has not received RTP packet of other nodes within the time $T_{l}$, then check its node cache space whether forward the data packet.

2) If not be mediated grouping, the node back to sleep state; the nodes can avoid collisions and save energy in the sleep state, the less the residual energy of nodes as the smaller the probability of the forwarding nodes. Here, giving under the adapt sleep mechanism of the node $N_{i}$ of sleep duration time $T S_{i}$ of calculation formula is:

$$
T S_{i}=\frac{\lambda}{E_{i}}
$$

where $r$ shows the balance factor, $E_{i}$ shows residual energy of node $N_{i}$.

3) If it needs to be forwarded grouping, then the node monitor data channel and busy tone channel whether is idle. If not free, the node into sleep; if free, the nodes into the forwarding state, and send the RTP packet request forwarding.

a) If the node receives only a CTP response group, then the node will forward all cache data grouping.

b) If the node does not receive the CTP response group, the node will continue to monitor the CTS group, if it still does not receive the CTP grouping in the following $n$ send time slot, then the node into sleep state.

c) If it receives multiple CTP response packets or noise signals in the same time slot, sending the collision of message, then continue to monitor the CTP response packets.

4). If the node gets data channel and allow sending data packet, the first jump forward from the previous routing to receive all data packet, then forward packet by itself. Which can increase the success probability of data packet arrived at the receiving node, and avoid the previous routing node energy waste.

\section{PROTOCOL PERFORMANCE ANALYSIS}

This paper put forward the energy efficient protocol based on the adaptive mechanism of sleep, at the same time considering the node residual energy and location information of the receiving node, implement the data packet forwarding, which can guarantee prolong the working life of the network under the receiving node throughput situation. We adopt OPNET10.0 simulation compare GERAF protocol and E2AS protocol of energy efficiency and throughput size. Among them, the parameter is set to: network coverage area is $5 \mathrm{~km} \times 5 \mathrm{~km}$, the node number is 60 , each node of the initial energy for $200 \mathrm{~J}$.

In the following Fig. (2) shows the same intensity and load cases, GERAF protocol and E2AS protocol comparative analysis of energy consumption. It can be seen from the Fig. (2), the cluster head nodes in the GERAF protocol consumes more energy than leaf node, and the energy consumed more rapidly. Which makes the network need to select the next round of cluster head nodes, and the next round of cluster head nodes also will soon run out of energy, until the entire network will not work. Cluster head nodes and leaf nodes in the E2AS protocol almost identical to the consumption, the overall network energy consumption more balanced, so as to prolong the network lifetime.

Fig. (3) shows under the same load condition, the receiving node throughput size comparison analysis under the GERAF protocol and E2AS protocol. It can be seen from the Fig. (3), the initial work in the network, because the GERAF protocol only considers node geographic location information, the selected route is the nearest node to the receiving node, and the receiving node throughput is larger at this stage. But with each round of cluster head nodes life terminated, network performance fall sharply, the receiving node throughput also fall sharply, until the entire network unable 


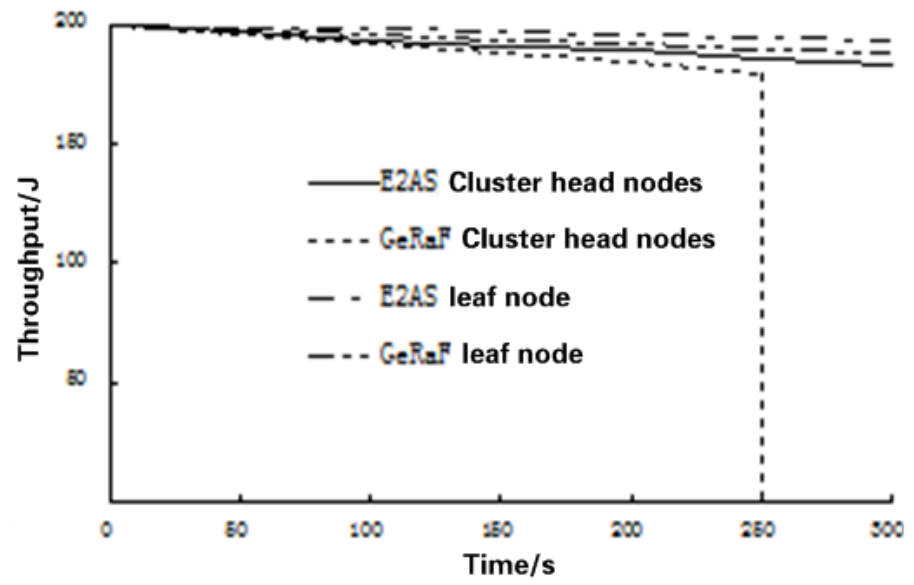

Fig. (2). GERAF protocol and E2AS protocol of residual energy comparison.

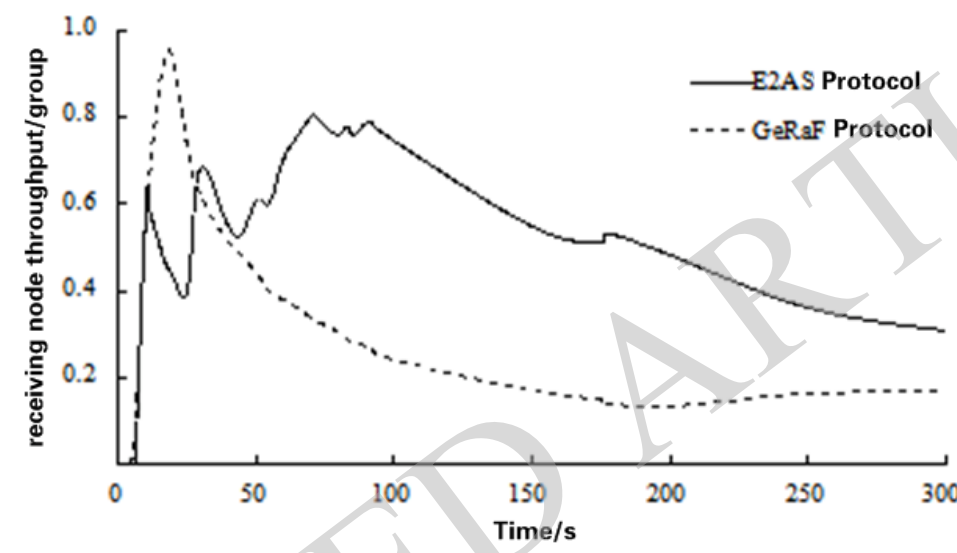

Fig. (3). Under the GERAF protocol and E2AS protocol receiving node throughput size.

to maintain normal work. But the E2AS protocol considered network node remaining stored energy and geographic location information at the same time, the receiving node of the throughput of the little change in a long period of time, increase the size of the receiving node throughput gently down over time.

\section{CONCLUSION}

Energy efficiency is the primary design goal and challenge in the wireless sensor network. This paper put forward the wireless sensor network energy efficient protocol which is combination of media access control protocol and routing protocol based on the adaptive mechanism of sleep, is a kind of cross-layer wireless sensor network protocol. Which can reduce the energy consumption of sensor nodes effectively and balance the network energy consumption at the same time, by setting the variable duration of sleep and choose the best forwarding nodes, and the aim of prolonging the service life of the network can be achieved. Protocol performance analysis results show that the E2AS protocol can be applied to a high network load intensive in the wireless sensor network, and has better theoretical research value and practical application value.

\section{CONFLICT OF INTEREST}

The authors confirm that this article content has no conflict of interest.

\section{ACKNOWLEDGEMENTS}

This work was financially supported by Special Fund of XJPCC Youth Science and Technology Innovation (2013CB009, 2014CB004) and Key Technology Developing Program of Shihezi University (gxjs2012-zdgg03).

\section{REFERENCES}

[1] H. P. Xie, H. Y. Zhou, and D. C. Zuo, "Wireless sensor network energy optimization and modeling techniques were reviewed," Computer Science, vol. 39, no. 10, pp. 15-20, 2012.

[2] J. Le, W. M. Zhang, W. D. Xiao, "An energy efficient and balanced wireless sensor network clustering data fusion algorithm," Journal of National University of Defense Technology, vol. 34, no. 6, pp. 6671, 2012.

[3] L. M. Sun, J. Z. Li, and Y. Chen, Wireless Sensor Network, Beijing: Tsinghua University Press, pp. 4-7, 2005.

[4] T. Liu, X. F. Wang, and J. Yan, "Based on the relative distance of multistage energy in the heterogeneous sensor network clusters algorithm," Computer Science, vol. 39, no. 8, pp. 119-125, 2012.

[5] D. Y. Gao, L. J. Zhang, and H. C. Wang, "Energy saving with node sleep and power control mechanisms for wireless sensor networks," The Journal of China Universities of Posts and Telecommunications, vol. 18, no. 1, pp. 49-59, 2011.

[6] H. W. Tang, J. N. Cao, X. F. Liu, and C. X Sun, "SR-MAC: A low latency MAC protocol for multi-packet transmissions in wireless sensor networks," Journal of Computer Science and Technology, vol. 28, no. 2, pp. 329-342, 2013.

[7] J. 1. Lu, and X. M. Wang, "Interference-aware probabilistic routing for wireless sensor networks," Tsinghua Science and Technology, vol. 17, no. 5, pp. 575-585, 2012.

[8] Z. Liu, and H. Guo, "Clustering network model based on the concentric ring hollow WSN energy avoid method research," Computer Science, vol. 40, no. 12, pp. 147-151, 2013. 
[9] G. Y. Lu, L. F. Wan, and S. F. Mi, "Wireless sensor network energy optimization research reviewed," Journal of Dalian institute for Nationalities, vol. 15, no. 3, pp. 309-313, 2013.

[10] F. N. Bouabdallah, and R. Boutaba, "Load-Balanced routing scheme for energy-efficient wireless sensor networks," Proceed- ings of IEEE Global Telecommunications Conference, pp. 1-6, 2008.

[11] M. Zorzi, and R. R. Rao, "Geographic random forwarding (GERAF) for Ad Hoc and sensor networks: energy and latency performance," IEEE Transaction on Mobile Computing, vol. 2, no. 4, pp. 349-365, 2003.

Received: September 16, 2014

Revised: December 23, 2014

Accepted: December 31, 2014

(C) Changzheng and Ronghua; Licensee Bentham Open.

This is an open access article licensed under the terms of the Creative Commons Attribution Non-Commercial License (http://creativecommons.org/licenses/by-nc/3.0/) which permits unrestricted, non-commercial use, distribution and reproduction in any medium, provided the work is properly cited. 